\title{
Sensitivity and Specificity of Pre-Operative Staging of the Axilla When Using OSNA in Breast Cancer Patients
}

\author{
Jonathan D. Horsnell1*, Kashif Choudhry' ${ }^{1}$, Daniel Urriza-Rodriguez ${ }^{1}$, Alex Valencia ${ }^{2}$, \\ Zenon Rayter ${ }^{1}$ \\ ${ }^{1}$ Department of Breast Surgery, North Bristol NHS Trust, Bristol, England \\ ${ }^{2}$ Department of Radiology, North Bristol NHS Trust, Bristol, England \\ Email: "jonathanhorsnell@hotmail.com
}

Received 15 April 2016; accepted 25 June 2016; published 28 June 2016

Copyright (C 2016 by authors and Scientific Research Publishing Inc.

This work is licensed under the Creative Commons Attribution International License (CC BY).

http://creativecommons.org/licenses/by/4.0/

(c) (i) 0pen Access

\section{Abstract}

Introduction: All patients with a new diagnosis of breast cancer undergo pre-operative axillary ultrasound (US) and biopsy to identify axillary metastases. The efficacy of this approach at identifying metastatic disease is particularly important if an intra-operative technique of assessment is used. This paper is the first to report the sensitivity and specificity of pre-operative axillary ultrasound within the context of the routine use of OSNA (one step nucleic acid amplification) (Sysmex, Japan), a molecular assay technique for the intra-operative analysis of axillary lymph nodes. This knowledge will help counsel patients prior to the procedure and inform economic analysis of the implications of this approach. Method: Consecutive patients diagnosed with invasive breast cancer between July 2009 and February 2012 at a single centre were identified by interrogation of MDT (multi disciplinary team) and surgical databases and a prospective database of all patients undergoing OSNA analysis. A standardised data collection sheet was used to record tumour characteristics and the nodal stage for each patient. These results were used to document the efficacy of preoperative US and biopsy of the axilla in comparison to intraoperative OSNA analysis. The data were then compared between two groups of patients, those whose nodes had been assessed using OSNA (the OSNA group) and those nodes had been tested using standard histopathological examination (the non-OSNA group). Results: A total of 377 eligible patients were identified. Within the OSNA group $(n=240)$ the sensitivity of pre-operative axillary US was $29 \%$ which rose to $41 \%$ when micro-metastases were excluded. A specificity and positive predictive value of $100 \%$ was recorded. The negative predictive value was $74 \%$. There were no significant differences to the efficacy when the nodes were assessed with standard histopathology. The detection rate of micro-metastases was significantly higher in the OSNA group $(P=0.001)$, but there was no statistically significant difference in those with macro-metastases $(P=0.457)$. Conclusions: There are no

\footnotetext{
Corresponding author.
}

How to cite this paper: Horsnell, J.D., Choudhry, K., Urriza-Rodriguez, D., Valencia, A. and Rayter, Z. (2016) Sensitivity and Specificity of Pre-Operative Staging of the Axilla When Using OSNA in Breast Cancer Patients. Advances in Breast Cancer Research, 5, 103-110. http://dx.doi.org/10.4236/abcr.2016.53012 
significant differences in the sensitivity and specificity achieved by pre-operative axillary US when compared to either post operative histopathology or OSNA molecular analysis as the gold standard. OSNA, however, does increase the detection of micro-metastases.

\title{
Keywords
}

\author{
Breast Cancer, Axillary Metastases, Ultrasound, OSNA
}

\section{Introduction}

The assessment of axillary lymph nodes in patients newly diagnosed with breast cancer is a key factor in assessing prognosis and helps guide treatment options and disease management for the individual patient [1]. Guidelines within the United Kingdom recommend that all patients with a new diagnosis of breast cancer undergo ultrasound (US) examination of their axillae with a guided biopsy of any suspicious nodes prior to their primary surgery [2]. Sentinel lymph node biopsy (SLNB) has become the standard of care for the analysis of patients with a clinical and ultrasound negative axilla whilst an axillary clearance remains the favoured approach for those patients found to have a positive node pre-operatively. The sensitivity of US, in this context, has been reported to range from $25 \%$ - $63 \%$ with specificities of up to $100 \%$ [1]. These results are based on the comparison of ultrasound to traditional gold standard post-operative histopathological assessment of the sentinel nodes.

In some centres the use of intra-operative methods of lymph node assessment is favoured. The advantages of intra-operative lymph node assessment include a reduction in patient anxiety, the avoidance of a second operative procedure if the nodes are positive, and a subsequent reduction in the use of healthcare resources [3] [4]. A number of techniques have been used in this setting. These include frozen section analysis, touch imprint cytology and molecular analysis. The sensitivity and specificities of these techniques vary with $57 \%-74 \%$ sensitivity and 100\% specificities reported for frozen section [3] [5], 63\% sensitivity and 99\% specificity for touch imprint cytology [6] and sensitivities of between $95 \%$ - 98\% with specificities of up to $97 \%$ for molecular assays [6]. Tests which utilise molecular assays use either reverse transcriptase polymerase chain reactions (RT-PCR) or reverse transcriptase loop-mediated isothermal amplifications (RT-LAMP). These have been developed to detect metastasis by quantitatively measuring the concentration of tumour specific mRNA markers. One of the distinct differences between the molecular assays and traditional pathological techniques is that the whole node is homogenised as part of the process. As a result post-operative histopathological assessment cannot take place. Further it is possible that small metastases not previously identified by standard histopathology may be detected. Previous work has demonstrated that increasing interrogation of lymph nodes, histologically, using a $2 \mathrm{~mm}$ step sectioning protocol upstaged between $7 \%$ and $42 \%$ of cases when compared to a single section and that the use of OSNA increases the rate of detection of micro-metastases [7] [8].

A disadvantage of the use of intra-operative testing is that it can add extra time to the operation. This might occur due to a wait for the test result or because of the extra surgery needed (an axillary clearance) if the node is found to harbour metastatic disease. Previous work has demonstrated that waiting for the results may add up to 20 minutes to the length of a wide local excision and sentinel lymph node biopsy with further added time if an axillary clearance is required [9]. This could significantly impinge on the planning and completion of the operating list. Identification of patients with axillary disease pre-operatively helps reduce this risk, thus an appreciation of the efficacy of pre-operative ultrasound of the axillae is vital. All previous work has used histopathological assessment of the node as the gold standard.

Within the breast surgery unit at our institution a protocol has been adopted that includes the use of both preoperative US followed by either fine needle aspiration (FNA) or core biopsy and intra-operative OSNA (Sysmex, Japan) assessment of any nodes that are clinically and radiologically negative. This is the only molecular assay currently available commercially. It has been adopted by a number of UK centres, including our own and has recently been approved for use by the National Institute for Clinical Excellence (NICE) in the UK [9] [10]. The technique utilises the RT-LAMP methodology to quantify the expression of cytokeratin-19 (CK-19) within the axillary nodes [11]. The impact that this has on the sensitivity and specificity of pre-operative ultrasound examination of the axillae has not been previously explored and this paper is the first to document the impact 
that the use of OSNA has in this context.

The primary aim of this study was to report the efficacy of pre-operative US assessment of the axillae when using OSNA as the gold standard comparison. Secondary outcomes included a comparison of these results to those achieved using standard post-operative histopathological testing. Further this would allow the incidence of macro-metastases and micro-metastases to be compared in these two scenarios for a consecutive series of patients.

\section{Methods}

A prospective database of consecutive patients, the "OSNA group" diagnosed with primary breast cancer within our institution was created for all patients undergoing sentinel node biopsy and OSNA lymph node analysis (July 2010 to February 2012). A retrospective case note and electronic systems review was performed to create a second database, the "non-OSNA group" of all primary breast cancer patients who underwent surgery for a year prior to the full implementation of sentinel node biopsy and intra-operative testing (July 2009 to July 2010).

All patients who underwent an ultrasound at the time of initial diagnosis and subsequent axillary clearance were identified based on multi-disciplinary team (MDT) databases, radiological reporting systems, operating records and diaries. At the time of initial presentation all patients underwent clinical breast examination, radiological investigation and either a core biopsy or FNA of any suspicious areas within the context of a one stop clinic at a single university teaching hospital. For any patient in which there was a diagnosis of breast cancer an ultrasound of the axilla was performed by a consultant radiologist, with an FNA or core biopsy of any abnormal nodes. If, on examination by a consultant pathologist, there was evidence of metastatic disease then the patient underwent an axillary clearance as their primary axillary surgery. If the node was negative on ultrasound with or without biopsy then the patient underwent a sentinel lymph node biopsy. For patients undergoing surgery before full OSNA implementation, post-operative histopathological assessment was performed. For all patients presenting after July 2010 intra-operative OSNA assessment at the time of their primary surgery was carried out. All patients who received neo-adjuvant treatment were excluded from the study.

Patients having a SLNB attended the nuclear medicine department not more than 24 hours prior to surgery for a sub-dermal injection of a radioactive isotope (Nanocoll, G.E. Healthcare, Chicago, USA). Immediately prior to surgery each patient received a peri-areolar injection of $2 \mathrm{ml}$ of $2.5 \%$ Patent V Blue dye (Guerbet, Roissy, France) based on the position of their tumour, followed by 5 minutes of breast massage. A gamma probe was then employed to guide the position of the incision and subsequent dissection of the sentinel lymph nodes. Following excision all radioactive and blue nodes were denuded of surrounding fat and either submitted for histopathological analysis or sent for intra-operative analysis using OSNA. The OSNA assay was performed in a separate room adjacent to the operating theatre. The SLNB was performed immediately prior to the breast surgery, which continued whilst the OSNA test took place. The results were then conveyed to the operating surgeon prior to the patient being woken from anaesthesia. If the node was negative (copy number $<250 \mu \mathrm{L}$ CK19 mRNA) then no further axillary surgery was performed. If the node was positive for macro-metastases (copy number $>5000 \mu \mathrm{L}$ ) or micro-metastases (copy numbers $250-5000 \mu \mathrm{L}$ ) then an immediate axillary clearance was performed and the further nodes excised were sent for histopathological assessment. All patients were consented for this possibility. At the start of the study the finding of micro-metastases was followed by an immediate axillary clearance. However following local audit, supported by the results of the IBCSG 23-01 trial and the recent St Gallen consensus [12] [13], this practice was discontinued and only patients positive for macro-metastases went on to have an axillary clearance.

Statistical analysis was performed using GraphPad software (GraphPad Inc., La Jolla, USA). A t-test was used for continuous data, and either a Fishers exact test or Chi squared test was used for categorical data. Details of which test were used are given in the tables that present the data.

\section{Results}

276 patients with a new diagnosis of primary invasive breast cancer were included in the prospective database of all patients undergoing SLNB and OSNA. 36 were excluded from this study as they had received neo-adjuvant chemotherapy, thus 240 patients were included in the "OSNA group". 165 patients who had undergone SLNB without intra-operative testing were identified by retrospective review. 28 of these patients were excluded from this study as they had either received neo-adjuvant chemotherapy $(n=9)$ or had not received a pre operative axillary ultrasound $(n=19)$. A total of 137 patients were therefore included in the "non-OSNA" group. 


\subsection{Patient Characteristics}

The patients in each of these groups were not selected but represented a consecutive series of patients presenting to the department. As expected there were no significant differences in the age, tumour grade or size in either group (Table 1).

\subsection{Sensitivity and Specificity of Pre-Operative Ultrasound Scans}

23 patients $(10 \%)$ in the OSNA group had a positive lymph node identified pre-operatively using axillary ultrasound and biopsy. All of these patients were found to have macro metastases at axillary clearance. Of the remaining 217 patients undergoing a SLNB, 161 (74\%) had a negative axilla and $56(26 \%)$ had evidence of positive sentinel nodes. Of these 56 patients 23 had micro-metastases and 33 had macro-metastases (Figure 1).

In comparison to OSNA as the gold standard the sensitivity of pre-operative ultrasound for identifying microand macro-metastases disease was $29 \%$ and for identifying macro-metastases was $41 \%$. The specificity and positive predictive value was $100 \%$, with a negative predictive value of $84 \%$ for macro-metastases and $74 \%$ for both micro- and macro-metastases (Table 2).

$9(7 \%)$ of the non-OSNA group had a positive axilla identified before surgery, all of which were found to have macro-metastases. Of the remaining 128 patients in this group $98(77 \%)$ had a negative axilla at subsequent

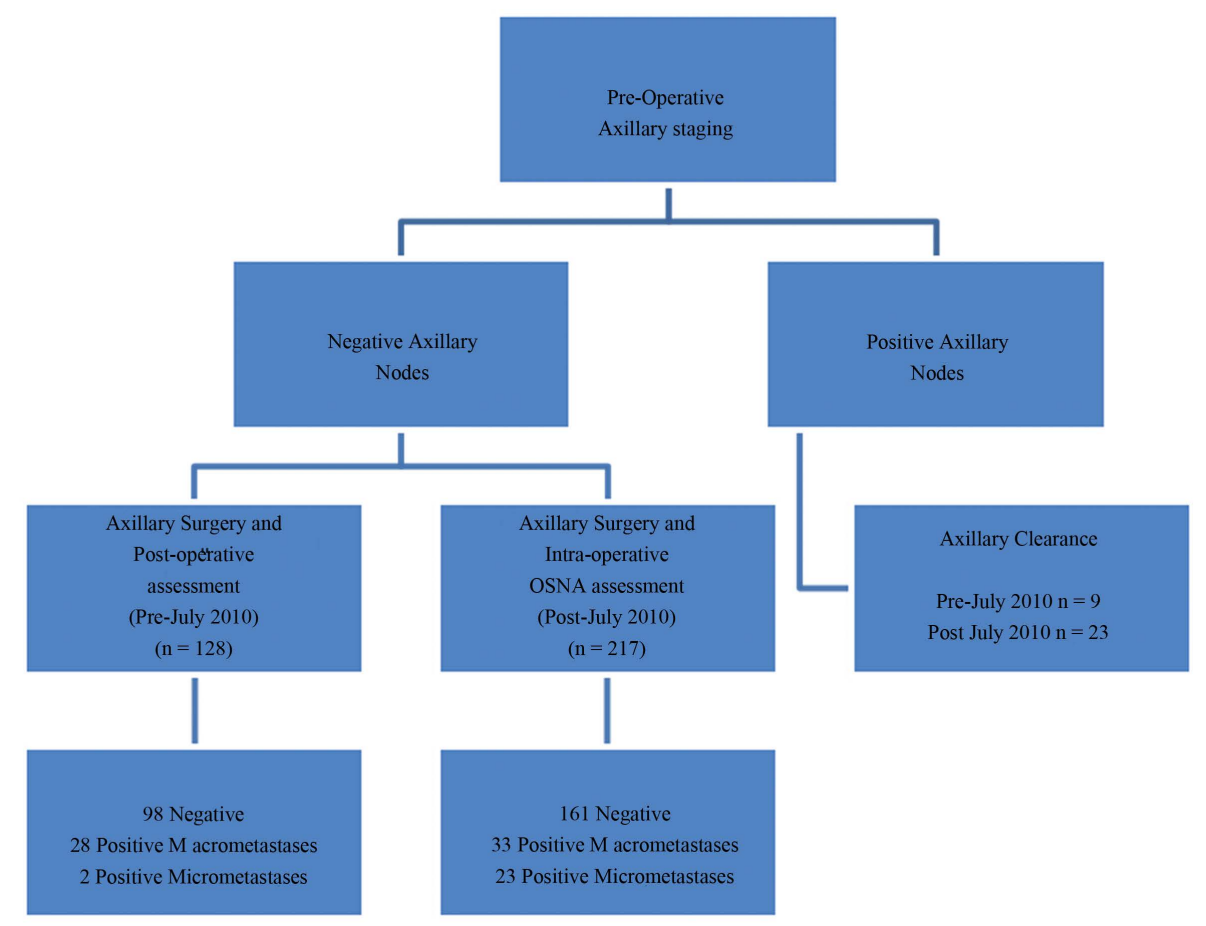

Figure 1. Flow chart demonstrating the outcomes of tests for patients with a new diagnosis of breast cancer that they underwent following their initial pre-operative ultrasound scan.

Table 1. Demographic details of the patients in each group demonstrating patients age, tumour grade and size and total number of involved nodes ( $\mathrm{SD}=$ standard deviation).

\begin{tabular}{cccc}
\hline & Non-OSNA Group $(\mathrm{n}=137)$ & OSNA Group $(\mathrm{n}=240)$ & \\
\hline 1) Age (mean in years +/- SD) & $60.8(+/-12)$ & $61.4(+/-12)$ & $\mathrm{P}=0.642^{*}$ \\
2) Tumour Grade (mode) & 2 & 2 & $\mathrm{P}=0.58^{* *}$ \\
3) Tumour Size (mean in mm +/- SD) & $18.9(+/-11.1)$ & $16.9(+/-10.7)$ & $\mathrm{P}=0.1^{*}$ \\
4) Total Nodes Involved (mean +/- SD) & $0.77(+/-2.5)($ range $0-23)$ & $0.86(+/-2.5)($ range $0-23)$ & $\mathrm{P}=0.74^{*}$ \\
\hline
\end{tabular}

*using unpaired t-test, ${ }^{* *}$ using Chi-squared test. 
Table 2. The sensitivity, specificity, positive predictive value (PPV) and negative predictive value (NPV) of US and FNA or core biopsy in comparison to OSNA and histopathology (for those proceeding direct to axillary dissection) at detecting all axillary metastases (micro and macro-metastases, Table $2 \mathrm{a}$ ) and macro-metastases alone (Table $2 \mathrm{~b}$ ) in patients undergoing surgery for primary breast cancer.

(a)

\begin{tabular}{lcccc}
\hline & & \multicolumn{2}{c}{ Final Status } & \\
\cline { 3 - 4 } & & Micro- or Macro-metastases & Negative \\
\hline \multirow{2}{*}{ Ultrasound FNA } & Micro- or Macro-metastases & 23 & 0 & PPV $=100 \%$ \\
& Negative & 56 & 161 & NPV $=74 \%$ \\
& Sensitivity 29\% & Specificity $100 \%$ & \\
\hline
\end{tabular}

(b)

\begin{tabular}{lcccc}
\hline & & \multicolumn{3}{c}{ Final Status } \\
\cline { 3 - 4 } & & Macro-metastases & Negative & \\
\hline \multirow{2}{*}{ Ultrasound FNA } & Macro-metastases & 23 & 0 & PPV $=100 \%$ \\
& Negative & 33 & 184 & NPV $=84 \%$ \\
& & Sensitivity $41 \%$ & Specificity $100 \%$ & \\
\hline
\end{tabular}

primary axillary surgery and $30(23 \%)$ had at least one positive node identified. Of those 30 patients, 28 had macro metastases and 2 had micro-metastases (Figure 1).

In comparison to standard histopathology assessment of the nodes as the gold standard, the sensitivity of pre-operative ultrasound was $23 \%$ for both micro and macro metastases, and $29 \%$ for macro metastases alone. The specificity and positive predictive value was $100 \%$ with a negative predictive value of $79 \%$ for macro-metastases (Table 3).

\subsection{Comparison between the Two Groups}

\subsubsection{Effectiveness of Pre-Operative Ultrasound Scan and Biopsy}

A Fishers exact test was used to compare the effectiveness of pre-operative US and biopsy when compared to either OSNA or post operative histopathology as the gold standard. There were no statistically significant differences in the sensitivity for patients with positive nodes (including micro-metastases and macro-metastases) or the sensitivity for macro-metastases alone. Further the differences in the specificity, positive predictive values, and negative predicative values were also not statistically significant (Table 4).

\subsubsection{Final Axillary Status}

There were no statistically significant difference between the two groups in the number of patients diagnosed with metastatic disease prior to surgery (23/240 in the OSNA Group vs. 9/137 in the non-OSNA group P =0.34). When comparing the final status of the axilla, there was a higher rate of micro-metastases in the OSNA group in comparison to that picked up by standard histopathology in the non-OSNA group $(23 / 230 \mathrm{vs.} 2 / 137)$ which was statistically significant $(P=0.001$, Fishers exact test). Amongst those who were US negative there were more patients with macro-metastases in the non-OSNA group which demonstrated a trend towards significance $(33 / 217$ vs $28 / 128, \mathrm{P}=0.14)$ (Table 5).

\section{Discussions}

The use of SLNB has become the standard of care for patients with a new diagnosis of breast cancer. Identification of lymph node metastases prior to surgery can allow for an immediate axillary clearance to take place without the need for a SLNB first. The primary aim of this study was to document the sensitivity, specificity and the positive and negative predictive values of pre-operative US and biopsy within the context of OSNA use. These results confirm the efficacy of this approach with no significant differences between the results obtained when using OSNA or post operative histopathology as the gold standard. An understanding of these results 
Table 3. The sensitivity, specificity, positive predictive value (PPV) and negative predictive value (NPV) of US and FNA or core biopsy at detecting all axillary metastases (micro and macro-metastases, Table 3a) and macro-metastases only (table $3 b$ ) in patients undergoing surgery for primary breast cancer in comparison to histopathology.

(a)

\begin{tabular}{lcccc}
\hline & & \multicolumn{2}{c}{ Post Operative Histopathology } & \\
\cline { 3 - 4 } & & Macro-metastases & Negative & \\
Ultrasound FNA & Micro- or Macro-metastases & 9 & 0 & PPV $=100 \%$ \\
& Negative & 30 & 98 & NPV $=77 \%$ \\
& Micro- or Macro-metastases & 9 & \\
\hline
\end{tabular}

(b)

\begin{tabular}{lcccc}
\hline & \multicolumn{3}{c}{ Post Operative Histopathology } \\
\cline { 3 - 4 } & Macro-metastases & Macro-metastases) & Negative & \\
\hline \multirow{2}{*}{ Ultrasound FNA } & Negative & 28 & 0 & PPV $=100 \%$ \\
& & Sensitivity 26\% & NPV $=78 \%$ \\
& & Specificity $100 \%$ & \\
\hline
\end{tabular}

Table 4. A comparison of the sensitivity, specificity, positive predictive value (PPV) and negative predictive value (NPV) achieved by pre-operative US and FNA or core biopsy when compared to either histopathology or intra-operative OSNA analysis and histopathology (for those who had pre-operative positive nodes) in the two groups of patients.

\begin{tabular}{|c|c|c|c|}
\hline & Non-OSNA group & OSNA group & (All Fishers Exact Test) \\
\hline \multicolumn{4}{|c|}{ Micro- and Macro-metastases } \\
\hline Sensitivity & $23 \%$ & $29 \%$ & $\mathrm{P}=0.82$ \\
\hline Specificity & $100 \%$ & $100 \%$ & $\mathrm{P}=1$ \\
\hline Positive Predictive Value & $100 \%$ & $100 \%$ & $\mathrm{P}=1$ \\
\hline \multirow[t]{2}{*}{ Negative Predictive Value } & $77 \%$ & $74 \%$ & $\mathrm{P}=0.7$ \\
\hline & Non-OSNA group & OSNA group & \\
\hline \multicolumn{4}{|c|}{ Macro-metastases only } \\
\hline Sensitivity & $26 \%$ & $41 \%$ & $\mathrm{P}=0.12$ \\
\hline Specificity & $100 \%$ & $100 \%$ & $\mathrm{P}=1$ \\
\hline Positive Predictive Value & $100 \%$ & $100 \%$ & $\mathrm{P}=1$ \\
\hline Negative Predictive Value & $78 \%$ & $84 \%$ & $\mathrm{P}=0.14$ \\
\hline
\end{tabular}

which show that 1 in 7 patients with a negative axillae will go on to have a macro metastases can help counsel patients undergoing this procedure and inform planning of the operating list. Further this information can be used to support the economic modelling of the impact of OSNA use without relying on the extrapolation of data from patients who did not undergo OSNA testing. Our secondary outcomes confirm the increased detection rate of micro metastases when using OSNA, disease which may not have been identified using standard histopathological testing. The omission of further surgery for these patients is thus warranted given that there has now been a strong move away from clearing for micro metastases identified by standard techniques.

The sensitivities reported here for the detection of both micro and macro-metastases (23\% to $29 \%$ ) are at the lower end of those previously published [14] [15]. This may reflect the fact that the dataset includes micro-metastases within the positive node group. Indeed when the sensitivity for macro-metastases alone is calculated the sensitivity increases from $29 \%$ to $41 \%$ in the OSNA group. Further, patients who received neo-adjuvant chemotherapy were excluded from this analysis. This group of patients, often with more aggressive disease, has a higher burden of disease within the axilla. If the data from this group had been included then 
Table 5. Axillary status of patients in the two groups. Results of initial US and FNA or core biopsy and subsequent primary axillary surgery if the US was negative.Overall axillary status combining the results of the pre-operative US and either FNA or core biopsy, primary axillary surgery and axillary clearance (if performed).

\begin{tabular}{cccc}
\hline & Non-OSNA group & OSNA group & (Fishers exact test) \\
\hline Pegative & Pre-operative Axillary Status & \\
Positive & 128 & 217 & $\mathrm{P}=0.34$ \\
\hline Regative & 9 & 23 & $\mathrm{P}=0.70$ \\
\hline Macro-metastases & 98 & 161 & $\mathrm{P}=0.14$ \\
Micro-metastases & 28 & 33 & $\mathrm{P}=0.001$ \\
\hline Negative & 2 & 23 & $\mathrm{P}=0.42$ \\
Positive & Overall Axillary Status & 161 & $\mathrm{P}=0.457$ \\
\hline
\end{tabular}

the sensitivity at detecting macro-metastases in the OSNA group would have increased further to $51 \%$.

When considering the secondary outcomes, a limitation of this study is that it compares patients over two time periods and thus is open to potential bias because of changes in practice during that time. There was a trend towards significance in the sensitivity and negative predictive value for the detection of macro-metastases in the OSNA group. This may be the result of an improvement in technique and experience at the later end of the study period. One of the key factors in this may relate to the increased use of core biopsy rather than FNA as confidence with the technique increased. This improvement in pre-operative detection may also account for the increased rate of SLN macro-metastases in the non-OSNA group. This is supported by the similar overall macro-metastases rate with the two groups. The use of neo-adjuvant treatments increased over the study period (5\% non-OSNA group and $13 \%$ OSNA group, $\mathrm{P}=0.006$ ). These patients were excluded from analysis because of the variability in the timing of the sentinel lymph node biopsy. However, as a result patients with a higher burden of disease were more likely to be excluded from the OSNA group and this may account for the increased tumour size that tends towards significance as patients with larger tumours may be more likely to undergo neo-adjuvant treatment.

This study has documented for the first time the sensitivity and specificity that pre-operative interrogation of the axillary nodes using US and biopsy can achieve when using intraoperative OSNA assessment as the gold standard. Further within the limitations of this study, despite an increase in the number of patients found to have micro-metastases, it has been demonstrated that these sensitivities and specificities are comparable to those previously achieved using histopathology as the gold standard.

\section{Acknowledgements}

The authors would like to thank Miss A Chaudhry, Mr Jim Cook, Miss Zoe Winters and all the OSNA laboratory technicians for their help and support with this paper.

\section{Conflict of Interests}

All authors confirm that they have no conflicting interests that may have had an influence on the preparation of this paper.

\section{References}

[1] Alvarez, S., Anorbe, E., Alcorta, P., Lopez, F., Alonso, I. and Cortes, J. (2006) Role of Sonography in the Diagnosis of 
Axillary Lymph Node Metastases in Breast Cancer: A Systematic Review. American Journal of Roentgenology, 186, 1342-1348. http://dx.doi.org/10.2214/AJR.05.0936

[2] National Institute for Health and Clinical Excellence (2009) Early and Locally Advanced Breast Cancer. Clinical Guideline 80, National Institute for Health and Clinical Excellence, London WC1V 6NA.

[3] Tew, K., Irwig, L., Matthews, A., Crowe, P. and Macaskill, P. (2005) Meta-Analysis of Sentinel Node Imprint Cytology in Breast Cancer. British Journal of Surgery, 92, 1068-1080. http://dx.doi.org/10.1002/bjs.5139

[4] Creager, A.J. and Geisinger, K.R. (2002) Intraoperative Evaluation of Sentinel Lymph Nodes for Breast Carcinoma: Current Methodologies. Advances in Anatomic Pathology, 9, 233-243. http://dx.doi.org/10.1097/00125480-200207000-00003

[5] Snook, K.L., Layer, G.T., Jackson, P.A., de Vries, C.S., Shousha, S., Sinnett, H.D., Nigar, E., Singhal, H., Chia, Y., Cunnick, G. and Kissin, M.W. (2001) Multicentre Evaluation of Intraoperative Molecular Analysis of Sentinel Lymph Nodes in Breast Carcinoma. British Journal of Surgery, 98, 527-535.

[6] Layfield, D.M., Agrawal, A., Roche, H. and Cutress, R.I. (2011) Intraoperative Assessment of Sentinel Lymph Nodes in Breast Cancer. British Journal of Surgery, 98, 4-17. http://dx.doi.org/10.1002/bjs.7229

[7] Tan, L.K., Giri, D., Hummer, A.J., Panageas, K.S., Brogi, E., Norton, L., Hudis, C., Borgen, P.I. and Cody, H.S. (2008) Occult Axillary Node Metastases in Breast Cancer Are Prognostically Significant: Results in 368 Node-Negative Patients with 20-Year Follow-Up. Journal of Clinical Oncology, 26, 1803-1809. http://dx.doi.org/10.1200/JCO.2007.12.6425

[8] Leenders, M., Broeders, M., Croese, C., Richir, M., Go, H., Meijer, S., Langenhorst, B. and Schreurs, W. (2012) U1trasound and Fine Needle Aspiration Cytology of Axillary Lymph Nodes in Breast Cancer. To do or Not to Do? Breast, 21, 578-583. http://dx.doi.org/10.1016/j.breast.2012.05.008

[9] Cools-Lartigue, J., Sinclair, A., Trabulsi, N., Meguerditchian, A., Nesurolle, B., Fuhrer, R. and Meterissian, S. (2013) Preoperative Axillary Ultrasound and Fine-Needle Aspiration Biopsy in the Diagnosis of Axillary Metastases in Patients with Breast Cancer: Predictors of Accuracy and Future Implications. Annals of Surgical Oncology, 20, 819-827. http://dx.doi.org/10.1245/s10434-012-2609-7

[10] Castellano, I., Macrì, L., Deambrogio, C., Balmativola, D., Bussone, R., Ala, A., Coluccia, C. and Sapino, A. (2012) Reliability of Whole Sentinel Lymph Node Analysis by One-Step Nucleic Acid Amplification for Intraoperative Diagnosis of Breast Cancer Metastases. Annals of Surgery, 255, 334-342. http://dx.doi.org/10.1097/SLA.0b013e31823000ed

[11] Visser, M., Mehdi J., Horstman, A., Brink, A., Pol, R., van Diest, P., Snijders, P. and Meijer, C. (2008) Intra-Operative Rapid Diagnostic Method Based on CK19 mRNA Expression Node Metastases for the Detection of Lymph Node Metastases in Breast Cancer. International Journal of Cancer, 122, 2562-2567. http://dx.doi.org/10.1002/ijc.23451

[12] Chaudhry, A., Williams, S., Cook, J., Jenkins, M., Sohail, M., Calder, M., Winters, Z. and Rayter, Z. (2014) The Real-Time Intra-Operative Evaluation of Sentinel Lymph Nodes in Breast Cancer Patients Using One Step Nucleic Acid Amplification (OSNA) and Implications for Clinical Decision-Making. European Journal of Surgical Oncology, 40, 150-157. http://dx.doi.org/10.1016/j.ejso.2013.12.007

[13] (2013) DG8 Intra-Operative Tests (RD-100i OSNA System and Metasin Test) for Detection of Sentinel Lymph Node Metastases in Breast Cancer: Guidance. NICE Aug 2013, 7.

[14] Galimberti, V., Cole, B.F., Zurrida, S., et al. (2013) Axillary Dissection versus No Axillary Dissection in Patients with Sentinel-Node Micrometastases (IBCSG 23-01): A Phase 3 Randomised Controlled Trial. The Lancet Oncology, 14, 297-305. http://dx.doi.org/10.1016/S1470-2045(13)70035-4

[15] Goldhirsch1, A., Winer, E., Coates, A.S., Gelber, R.D., Piccart-Gebhart, M., Thürlimann, B. and Senn, H. (2013) Personalizing the Treatment of Women with Early Breast Cancer: Highlights of the St Gallen International Expert Consensus on the Primary Therapy of Early Breast Cancer 2013. Annals of Oncology, 24, 2206-2223. http://dx.doi.org/10.1093/annonc/mdt303 


\section{Submit or recommend next manuscript to SCIRP and we will provide best service for you:}

Accepting pre-submission inquiries through Email, Facebook, Linkedin, Twitter, etc A wide selection of journals (inclusive of 9 subjects, more than 200 journals)

Providing a 24-hour high-quality service

User-friendly online submission system

Fair and swift peer-review system

Efficient typesetting and proofreading procedure

Display of the result of downloads and visits, as well as the number of cited articles

Maximum dissemination of your research work

Submit your manuscript at: http://papersubmission.scirp.org/ 